\title{
The experiences of mothers who were implementing Kangaroo Mother Care (KMC) at a Regional Hospital in KwaZulu- Natal
}

\author{
J Reddy, M Cur \\ KwaZulu Natal College of Nursing
}

PA McInerney, PhD

School of Nursing, University of KwaZulu Natal

\section{Correspondence address:}

Dr. P. A. McInerney

School of Nursing

University of KwaZulu Natal

P.O. Dalbridge

4041.

Tel : (031) 260-2499

Fax : (031)260-1543

\section{Abstract: Curationis 30(3): $x-y$}

Kangaroo Mother Care (KMC) is a fairly new concept to the patients and personnel in the neonatal units in KwaZulu-Natal. Arising from one of the Governmental initiatives, $\mathrm{KMC}$ was introduced to KwaZulu Natal in 2001. The personnel at the regional hospital where this study took place, were briefly introduced to this alternate method of care for a low birth weight baby, by means of symposia and in-service training. This study was undertaken to explore the perceptions of mothers for the preparation and experience of KMC and to describe the experiences of the mothers who had implemented KMC. Furthermore, this study sought to determine whether mothers received support during the implementation of KMC and if so, to identify the sources of this support. The lack of documented evidence to problems that mothers may have encountered and management strategies to deal with these, prompted the study. A phenomenological approach was used. The sample was obtained from the neonatal unit of a regional hospital in Durban. The sample comprised of ten mothers who were practicing KMC in the post-natal ward, or mothers who were discharged and were still practicing KMC. Data were collected by means of face-to-face interviews. These interviews provided the researcher with rich, personal and narrative experiences of the carers before and during KMC. The results of this study indicated that KMC was indeed new to most of the mothers and this evoked apprehension, doubt and fear, but once the mothers had tried it and were successful, they felt a sense of joy. Nursing personnel formed part of the supportive environment for the mothers practicing KMC. Recommendations are made for nursing practice, nursing education and nursing research.

\section{Introduction}

In the past fifty years, regionalization of perinatal care, neonatal medicine, pediatric surgery, and diagnostic and therapeutic advances in obstetrics have increasingly contributed to the improved survival rates of low birth weight (LBW) and very low birth weight infants (VLBW). This improved survival has been at the price of separating the infant from the mother. Kangaroo Mother Care (KMC) a fairly new concept in KwaZulu
Natal reinstates the alienated mother to her rightful place in the management of her infant (Kirsten, Bergman and Hann, 2001:444). The term KMC is used to describe an infant being nursed in an upright position in skin-to- skin contact with the mother's breast. In the absence of the mother another carer viz. mother's partner, mother's friend, mother's sister or the baby's grandmother can substitute for the mother (Cattaneo, Davanzo, Worku, Surjono, Echeverria, Bedri, 
Haksari, Osorno, Gudetta, Setyowireni, Quintero \& Tumburlini, 1998:977).

The term KMC was first coined by Doctors Edgar Rey and Hector Martinez in 1978 in Bogota, Columbia (Kirsten et al 2001:444) and was first described in the English medical literature by Whitelaw and Sleath (1985:1206) following a visit to Bogota, Columbia. The Instituto Materno Infantil at San Juan de Dios Hospital is a very large maternity hospital where 11000 babies were delivered annually. Many of them are at high risk. The special care baby unit had always been overcrowded, underequipped and understaffed. Cross infection was common and the survival of very LBW infants was poor. Very large numbers of LBW babies with few incubators resulted in high mortality rates from infection. Partly as a means of getting babies discharged early, Rey and Martinez introduced maternal-infant skinto-skin contact if the infants survived the first week or two. The baby is dressed only in a small diaper and a woolen cap, then placed on the mother's naked chest. The baby can be secured in a number of ways. The simplest is to tie the baby on firmly with a non-stretching cotton cloth, for example a theatre towel. It should be knotted in the mother's axilla, to enable her to lie comfortably (Bergman and Jurisoo, 1994:57).

Rey and Martinez (Kirsten et al 2001:444) encouraged exclusive breast-feeding and discharged the babies early.

The Bogota Program was supported by the United Nations Children's Fund (UNICEF). In 1983 UNICEF reported that (Whitelaw and Sleath, 1985:1206) "Instead of being packed in an incubator, low birth weight babies are packed close to their mothers right next to the breast. The new technique needs no technology and is cost effective. Before the new techniques were introduced all babies weighing less than one kilogram died. Now three quarters of them are saved. For those weighing between 1000 and 1500 grams the death rates have dropped from $70 \%$ to $10 \%$ ". Whitelaw et al. (1988:1377) postulate that babies as small as 700 grams, who no longer require oxygen can safely be nursed in skin-toskin contact. However, in most of the world, early discharge is not a primary component of KMC. Skin-to-skin care and breast-feeding are the essential components of KMC (Charpak, RuizPelaez, Figueroa, and Charpak 1997:687)

\section{Problem Statement}

$\mathrm{KMC}$ is a new intervention for caring for LBW infants in KwaZulu Natal and was initiated by the Department of Health, KwaZulu Natal. Nursing staff received in-service education on KMC from the Department of Health by means of symposia and workshops, in preparation for this new intervention. With the support of the pediatricians, KMC was introduced in January 2002. Limited information was available to staff - only that which appeared in journal articles and research reports.

In South Africa the value of KMC has been studied at Groote Schuur Hospital in Cape Town and was reported by Hann, Malan, Kronson, Bergman and Huskisson (1999:39), and at Kalafong Hospital, near Pretoria (Van Rooyen, Pullen, Pattinson and Delport (2002: 6). Both of these studies focused on the effects of KMC in terms of the growth of the baby, the length of the hospital stay of mother and baby and the success of breast feeding. The study done at Groote Schuur Hospital, Cape Town, by Hann et al.(1999:37) was a controlled clinical trial with twenty eight low birth weight babies. More recently, van Rooyen et al (2002:6) have reported on the value of KMC at Kalafong Hospital. They report that 466 infants were admitted to the KMC unit over a period of 18 months. The average length of stay in the unit was 13 days and the average weight gain was $23 \mathrm{~g}$ per day. For infants weighing less than $1300 \mathrm{~g}$, the total length of hospital stay decreased by three days.

No studies were found that explored the experiences of the mothers in a South African context.

\section{Objectives of the Study}

These were to: -

- Determine and describe the
perceptions of mothers in the
preparation for KMC.
Describe the experiences of the
mothers who had implemented
KMC.
Determine whether mothers
received support during the
period of KMC implementation,
and
Identify the sources of support
for the mothers in KMC.

\section{Significance of the Study}

The advantages of KMC to the LBW and VLBW infant in terms of weight gain, length of hospital stay and breastfeeding has been well documented in a number of studies (Whitelaw and Sleath, 1985:1206; Bergman and Jurisoo, 1994: 57 : Charpak et al 1997:682 and Hann et al 1999:37).

KMC was not practiced in KwaZulu Natal (KZN) until January 2002. Since it is a fairly new concept in KZN, the experiences of mothers, who were implementing KMC was not known. With more research-based evidence on the experiences of mothers, nurses will be better equipped to plan a management protocol for mothers assigned to practice KMC.

\section{Definition of Concepts}

Low birth weight (LBW) - infants born with a birth weight of less than 2000 grams (Cattaneo et al. (1998:977), Sloan, Camacho, Rojas \& Stem (1994:782) \& Charpak et al (1997:682)).

Kangaroo mother care (KMC) - where the LBW infant is nursed in an upright position in skin- to- skin contact with the carer's chest. (Cattaneo et al. (1998:976), Sloan et al. (1994:782).

Conventional method of care (CMC) use of incubators and heated cribs to prevent hypothermia in LBW infants.

Stabilized - vital signs are within normal limits, not dependent on oxygen and/or intravenous fluids and no visible malformation/abnormality exists. (Cattaneo et al. (1998:977).

Experiences - knowledge or skill gained by personal observation or practical acquaintance with facts or events. ( Thompson,1996:304).

Perception - act on faculty of perceiving. Intuitive recognition of a truth. (Thompson, 1996:661).

\section{Research Approach}

The design used in this study was a qualitative, phenomenological approach. The population consisted of all mothers who had babies below 2000 grams and who had delivered at a regional hospital in KZN during the period February - June 2003.

\section{Sampling}

A purposeful theoretical sampling technique was used. The sample was drawn from the mothers whose babies 
had been admitted to the neonatal unit of the regional hospital and who subsequently went on to practice KMC. The eligibility criteria were that

the mother must have had a recent memory of the experience and therefore only those who had discontinued KMC two weeks previously were included in the study; the baby must have been in a stabile condition (i.e. the vital signs were within normal limits, was not dependent on oxygen and/or intravenous fluids and there was no visible malformation or abnormality); KMC had been adopted as the method of care for more than one week. The mothers had to be able to speak English. Sampling continued until saturation of data was achieved.

\section{Sample Size}

The sample comprised of ten mothers who met the above eligibility criteria. Mothers who were practicing KMC were approached by the staff in the neonatal unit and asked whether they would participate in the study. The names of these mothers were then referred to the researcher who then explained the purpose of the research to them, using an information sheet.

\section{Data Collection}

Data were collected over a five month period, by means of face-to-face interviews, by the researcher. Interviews were conducted in English. The interviews took place in a room attached to the ward in which mothers who were practicing $\mathrm{KMC}$ were nursed. Mothers were asked to describe their experiences in practicing $\mathrm{KMC}$. The researcher also used general probing questions such as:

"Can you tell me more about this? How did this make you feel?" to elicit more detail. The interviews were tape recorded. Due to the fact that many of the mothers practicing $\mathrm{KMC}$ were from remote areas, only one interview was conducted with each mother. On completion of the interview, the researcher verified the information given to her by summarizing each point and obtaining the mother's approval that information given had been correctly summarized.

\section{Data Analysis}

The recorded interviews were transcribed verbatim by the researcher. The researcher analyzed the transcribed interviews, using Morse's cognitive process of data analysis, by reading and re-reading the scripts to seek

Table 1 Themes and sub-themes identified

\begin{tabular}{|l|l|}
\hline Theme & Sub-theme \\
\hline Feelings & $\begin{array}{l}\text { Before KMC } \\
\text { During KMC }\end{array}$ \\
\hline Management of mothers & $\begin{array}{l}\text { By staff } \\
\text { By self } \\
\text { By others }\end{array}$ \\
\hline Support & $\begin{array}{l}\text { From health professionals } \\
\text { From other mothers } \\
\text { From family members }\end{array}$ \\
\hline
\end{tabular}

meaning in the data. Each line of the narrative was read and the text was divided into sections (Morse 1994:23). Each section was labelled with a code word or phrase that conveyed the meaning of the section. The codes with a common meaning were grouped into subthemes, which were in turn combined to form themes. An independent coder, who had experience in qualitative data analysis, was used to ensure trustworthiness. Analysis of the data was done manually.

\section{Trustworthiness}

The four criteria by which trustworthiness of the study was established were those advocated by Guba (de Vos 1998: 351). Credibility of the data was ensured by audio taping the interviews (Mouton 2001: 276). Dependability was ensured through the use of a more experienced second coder and confirmability was ensured through the use of participants' own words to describe phenomena. Bracketing of presuppositions of the researcher (credibility) and member checking (credibility) also contributed to trustworthiness.

\section{Ethical Considerations}

Written permission was sought and obtained from the University ethics committee, the KZN Department of Health, the medical superintendent and the deputy director of nursing at the regional hospital where the study was conducted. Written consent was also obtained from the mothers after they had been fully informed about the study, their right to refuse to participate in the study and of their right to withdraw from the study at any time. They were assured that their refusal to participate or their wish to withdraw would not in any way compromise their babies' care. Anonymity and confidentiality were ensured as names were not used during the interviews.

\section{Findings and discussion of findings}

The main themes that emerged from the interviews were feelings, management of mothers and support (See Table 1).

All participants interviewed wैere mothers. The majority of the mothers were between 20 and 25 years of age. Their educational level ranged between grades 8 to 12 .

\section{Themes}

\section{Feelings}

The sub-themes identified in relation to feelings were those feelings that were identified before commencing $\mathrm{KMC}$ and those that were identified during the implementation of KMC.

\section{Before KMC}

Mothers described their feelings before starting KMC as those of being afraid, anxious or confused. Some doubted that KMC would work.

One mother said:

"I was so afraid of KMC as it was the first time I was seeing it being done."

Another described her feelings as follows:

"At first I was so confused, I was just wondering how is this going to help my baby?"

Most of the mothers expressed their doubts about whether KMC would work - yes, I did have a doubt, I doubted whether KMC would work. 
Mothers were worried about their babies becoming hypothermic. One mother stated that although the nurses were monitoring the baby's temperature I was still afraid that the baby may get cold.

Other fears expressed by the mothers included whether this small baby would slide down the mother's chest and whether their baby would suffocate $-i$ was trying to push his head to one side and because of his nose I was afraid, I carried on putting his head on to one side. Another mother described how she supported her baby - initially when I put baby on me, I felt she is going to fall. I kept my hand under her all the time.

Feelings of apprehension regarding practising KMC have been reported by Neu (1999: 160). Whilst they had yearned to hold their babies, all the parents in this study, acknowledged being apprehensive, at least initially, when they held their infant in skin-to-skin contact. Bergman and Jurisoo (1994: 58) state that psychological support of the mother is essential. They stress the on going need to provide her with education, which must include all aspects of care. Temperature control, oxygen saturation and respiratory rates have been extensively researched in relation to $\mathrm{KMC}$. The findings in relation to temperature control are not consistent. Acolet, Sleath and Whitelaw (1989: 192) found that skin temperature and oxygen saturation were maintained during skin-to-skin contact. Bosque, Brady, Affonso and Wahlberg (1995: 219) however found a lower temperature in infants nursed in the Kangaroo position, whilst Bauer, Sontheimer, Fisher and Linderkamp (1996 : 610 ) report that skin temperature increased significantly during maternal and paternal Kangaroo care. Christensson, Siles, Moreno, Belaustequi, De La Fuente, Lagercrantz, Puol and Winburg (1992:1115) note that the maternal body is an efficient heat source for the LBW baby.

\section{During KMC}

Despite some apprehension being felt by the mothers prior to commencing $\mathrm{KMC}$, mothers acknowledged feelings of elation and excitement whilst practicing KMC. These feelings were perceived when the mothers began to witness the benefits of KMC. It was usually the baby's weight gain that triggered this response.

"The other patient's baby didn't gain weight, but when she practiced KMC the baby's weight just started going up and up. I saw the mother was so excited and when she returned to the KMC ward she told all the mothers that this KMC worked."

\section{"I was excited when I saw her gained weight."}

Parents' expressions of delight when seeing their babies respond to KMC has been reported by Gale, Franck and Lund (1993: 49). In their study parents expressed delight at watching their infants' oxygen saturation improve during holding.

In addition, to the feelings of excitement generated by the weight gain, feelings of emotional calmness and tranquility were also expressed. One mother said:

"Ah! The bond, the feeling. I can't explain how special it was."

Another mother said she can sleep for hours on me.

These findings are consistent with the literature. In Whitelaw et al's (1988: 1377) randomized control trial, it was found that babies with skin-to-skin contact were more tranquil. In Kambarami et al's study (2002: 132) mothers stated that their infants slept longer when on the chest and tended to cry more when they were put down. Ludington-Hoe and Swinth (1996: 697) note that the sleep state during Kangaroo care is deep, quiet and regular respiration sleep. They note further that Kangaroo care provides containment similar to in utero containment, thus evoking quiescence, decreased arousal, and a significant increase in the amount of quiet sleep.

Neu (1999:161) also reports that most parents described a special interaction that occurred between the parent and the infant during skin-to-skin contact. Parents described this feeling as intense connectedness. Gale et al (cited in Ludington-Hoe and Swinth 1996: 699) report that infants have been observed, extending their heads in an attempt to get into the en-face position with the parent providing Kangaroo care, gazing intently at the parent, and maintaining the gaze for exceptional lengths of time.

In addition to feelings of excitement and calmness, mothers also voiced feelings of determination and commitment whilst practicing $\mathrm{KMC}$.
I wanted to do what was good for my baby. I wanted my baby to gain weight. I was prepared to do anything for the sake of my baby's health.

Ludington Hoe et al (cited in Neu 1999:

162) report that mothers felt empowered by the skin-to-skin experience because it was their unique contribution to their infant's well being. Kambarami et al (2002: 132) report that mothers felt that KMC gave them opportunity to closely monitor the baby's condition and for early detection of any changes, such as colour, breathing or vomiting.

\section{Management of the Mothers}

This theme had three sub-themes management by staff, self and others.

Mothers felt that the nursing staff had been a pillar of strength to them. All the participants acknowledged receiving emotional and practical support on KMC before practicing it. Mothers received information in the form of a talk and a demonstration. The use of charts and pictures on KMC enhanced this education given to mothers. Mothers also stated that the nursing staff had helped them to attach their babies to their chests.

One mother stated, I wouldn't have gone through with it if it wasn't for the Sisters (registered midwives).

Mothers felt that they had coped with KMC. One mother had implemented KMC with one twin and she was not sure that she would have coped if she had had both twins on her chest.

Mothers acknowledged and described the encouragement that they had received from other mothers. Mothers encouraged one another with words such as:

"Don't be frightened, just be positive, it is very good. You will find that it works."

Bergman and Jurisoo $(1994 ; 58)$ have noted the importance of emotional and practical support from nursing staff, as well as the help of other mothers in the unit.

\section{Support}

Support was found to come from three main sources - health professionals, other mothers and family members.

In addition to the nursing staff, mothers stated that the medical staff and 
KAMBARAMI, RA; MUTAMBIRWA,J \& MARAMBA, PPP 2002: Caregivers' Perceptions and Experiences of 'kangaroo care' in a developing country. Tropical Doctor, 32(3) July 2002: 131 - 133.

KIRSTEN, GF; BERGMAN, NJ \& HANN, FM 2001: Kangaroo Mother Care in the Nursery. Paediatric Clinics of North America, 48(2)April 2001:443-452.

\section{LUDINGTON-HOE,SM\& SWINTH,JY}

1996: Developmental Aspects of Kangaroo Care. Journal of Obstetrics, Gynaecology \& Neonatal Nursing, 25(8) October 1996: 691 - 703 .

MOUTON, EBJ 2001: The Practice of Social Research. Cape Town: Oxford University Press.

MORSE, J M 1994: Emerging from the data: The cognitive process of analysis in qualitative inquiry. In MORSE J M (Ed). Critical Issues in Qualitative Research Methods. London: Thousand Oaks, Sage

NEU, M 1999: Parents Perception of Skinto-Skin Care with their Preterm requiring assisted ventilation. Journal of Obstetrics, Gynaecology \& Neonatal Nursing, 28 March/April 1999: 157- 163.

SLOAN, NL; CAMACHO, LWL; ROJAS, EP \& STERN, C 1994: Kangaroo mother method: randomized controlled trial of an alternative method of care for stabilized low birth weight infants. Lancet, 344 September 1994: 782 -785 .

THOMPSON, D 1996: The Pocket Oxford Dictionary. United Kingdom: Oxford University Press: 304 \& 661.

VAN ROOYEN, E; PUllen, A E; PATTINSON, R C \& DELPORT, S D 2002: The value of the Kangaroo Mother Care Unit at Kalafong Hospital. Geneeskunde - The Medical Journal April 2002: 6-10.

WHITELAW, A; HEISTERKAMP, G; SLEATH, K; ACOLET, D\& RICHARDS, $M$ 1988: Skin to Skin contact for very low birthweight infants and their mothers. Archives of Disease in Childhood, 63(11): 1377 - 1381.

WHITELAW, A \& SLEATH, K 1985: Myth of the Marsupial Mother : Home Care of very low Birthweight Babies in
Bogota, Colombia. Lancet, May 25 1985:1206-1208. 